\title{
América del Sur en el comercio global (2007-2016)
}

\author{
Milú O. V. Fernández Rodríguez*
}

\begin{abstract}
RESUMEN
El presente trabajo analiza las principales características del comercio de bienes y servicios de América del Sur ante los cambios en el contexto internacional. Principalmente, se analizan los flujos comerciales, los principales socios comerciales y las principales características de los bienes y servicios comercializados. Además, el análisis se realiza a nivel de región y país.
\end{abstract}

Palabras clave: América del Sur, comercio, bienes, servicios.

South America in global commerce (2007-2016)

\section{Abstract}

This paper analyzes the main characteristics of the trade of goods and services of South America according the changes in the international context. Mainly, it analyzes the commercial flows, the main commercial partners and the main characteristics of the goods and services marketed. In addition, the analysis is done at the region and country level.

Keywords: South America, Commerce, Goods, Services.

\section{Introducción}

En la última década, el contexto internacional ha pasado por fuertes cambios, lo cual ha impactado de diferentes formas en el flujo comercial y el papel que juega América del Sur en el comercio internacional.

La región aprovechó el crecimiento económico de China y otros países asiáticos, quienes demandaron crecientemente productos de la región como alimentos,

* Economista de la Pontificia Universidad Católica del Perú. Ha sido asistente de investigación y docencia en la misma casa de estudios. Actualmente, se desempeńa como asistente de la Gerencia Comercial y Nuevos Negocios en TGP Perú. Correo electrónico: milu.fernandez@pucp.pe 
minerales y petróleo. La mayor demanda provocó un alza histórica de los precios mencionados (Bartesaghi, 2013), lo cual implicó un aumento en el valor de las exportaciones debido a la importancia de dichos productos en la canasta exportadora sudamericana. Luego, la crisis internacional que comenzó en Estados Unidos y se extendió hacia la Unión Europea y la desaceleración económica china, impactó a la región provocando una desaceleración comercial a partir de 2012.

En este contexto, a continuación, se analiza las principales características del comercio de bienes y servicios de la región como la evolución del flujo comercial, la participación en el comercio mundial, los principales socios y las principales características de los bienes y servicios comercializados.

\section{Comercio de bienes}

Las exportaciones de América del Sur alcanzaron su mayor nivel en 2011, explicado principalmente por el auge de los precios básicos desde principios de la década de 2000, lo que contribuyo fuertemente al crecimiento de los valores de exportación (FMI, 2017). La tasa de crecimiento promedio de las exportaciones entre 2007-2011 fue de $17 \%$, a partir de dicho año las exportaciones se reducirían en promedio hasta en 2016 en 9\%. Las exportaciones en 2016 fueron 412682 millones de dólares, 38\% menos en comparación con lo exportado en 2011.

La reducción de las exportaciones a partir de 2012 se explica tanto por términos de precio como de volumen. La reducción de los precios de los productos básicos provocó una fuerte caída en el valor de las exportaciones. En 2012, las exportaciones se redujeron en 1,9\% producto de la reducción de los precios, mientras que, en 2014, implico una reducción del 6\% de las exportaciones (Cepal, 2013; Cepal, 2015). En términos de volumen, las exportaciones de la región también se han desacelerado, en consonancia con la débil demanda externa caracterizada principalmente por la desaceleración económica china. En 2011, las exportaciones aumentaron en $6 \%$ debido a la mayor demanda, mientras que al ańo siguiente solo impulso en $0,5 \%$ el crecimiento de las exportaciones (Cepal, 2012; Cepal, 2013).

En lo que respecta a las importaciones, la tasa de crecimiento entre 2007-2013 fue en promedio de $12 \%$, luego se reduciría el valor importado en promedio en $14 \%$. Las importaciones en el 2016 fueron 378290 millones de dólares, 14\% menos al año anterior. La reducción de las importaciones se debió a la menor demanda producida por la desaceleración económica de los países de la región.

Por otro lado, el saldo comercial sudamericano ha sido positivo para todos los años, a excepción de 2014 y 2015 donde la región presentó déficit comercial. Sin embargo, 
al analizar por países, se encuentra que Guyana, Paraguay y Uruguay han presentado déficit para todos los años entre 2007-2016, lo cual responde a la diferencia entre los valores agregados de los productos exportados e importados de dichos países.

\section{Gráfico 1}

América del Sur: comercio de bienes, 2007-2016

(En millones de dólares)

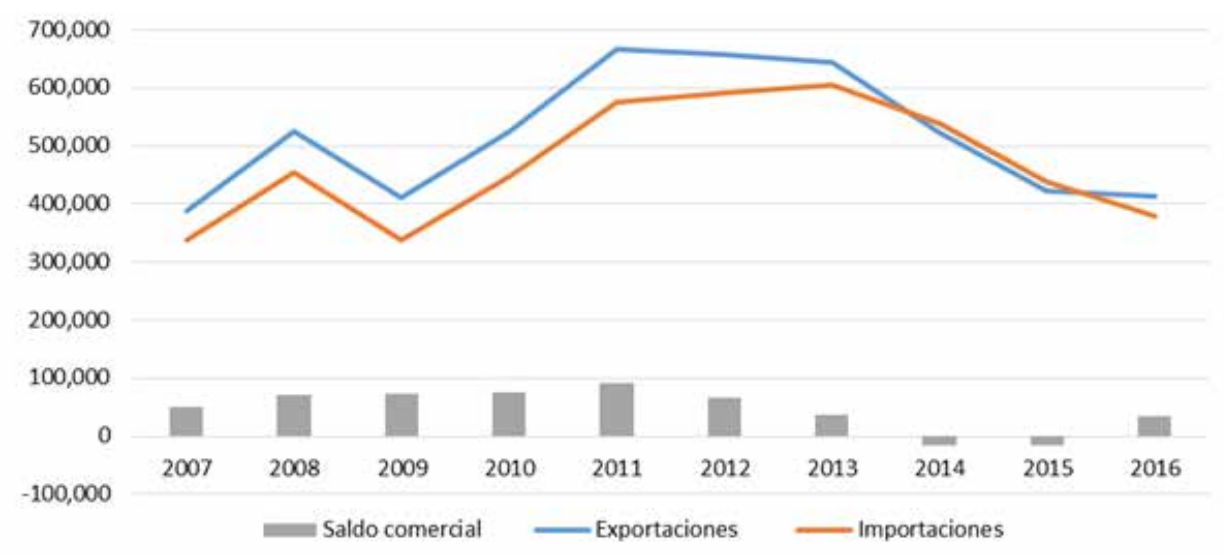

Fuente: Elaboración propia a partir de las estadísticas de COMTRADE y TRADEMAP.

Nota: Los datos de exportaciones e importaciones para Venezuela (2014-2016) y para Suriname (2015) no se encuentran disponibles.

En lo que respecta a la participación en el comercio internacional, las exportaciones entre 2007 y 2016 representaron en promedio el 3,2\% de las exportaciones mundiales, alcanzando mayor participación en 2011 con un 3,7\%. Mientras que las importaciones de la región representaron en promedio $2,9 \%$ del total de las importaciones mundiales para el periodo señalado, alcanzando su mayor participación en el 2013, con el 3,3\%.

$\mathrm{Al}$ analizar las exportaciones desde 2011, año donde la región alcanzó el mayor nivel de exportaciones, se encuentra que la tasa de variación promedio anual fue negativa para todos los países a excepción de Paraguay. Dentro de los países que tuvieron una mayor tasa negativa anual se encuentran Colombia $(-10,3 \%)$, Argentina $(-6,8 \%)$ y Brasil (-6,1\%).

Las exportaciones colombianas llegaron a su mayor nivel en 2012, a partir de dicho año se evidencia una fuerte reducción debido a las menores exportaciones mineroenergéticas que se redujeron en 63,3\% entre 2012-2016 (Vargas, 2017). En lo que respecta a las exportaciones argentinas, estas se redujeron a partir de 2012 hasta el 2015 principalmente por la menor exportación de manufacturas de origen industria, las cuales entre 2011-2015 disminuyeron en 38\%. Por otro lado, las exportaciones 


\section{Gráfico 2}

América del Sur: participación en el comercio internacional de bienes, 2007-2016

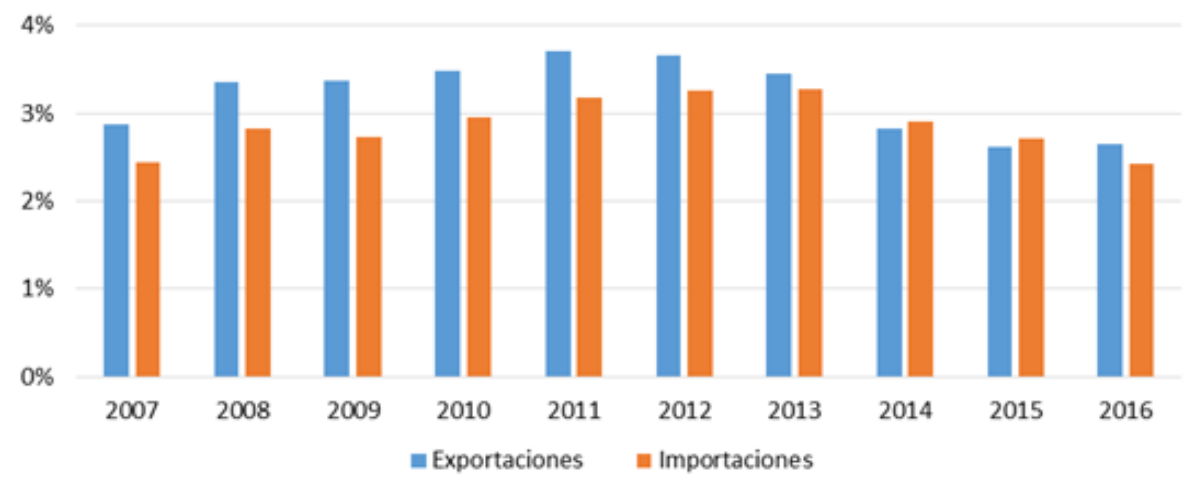

Fuente: Elaboración propia a partir de las estadísticas de COMTRADE y TRADEMAP.

brasileñas se redujeron a partir de 2012, principalmente por la reducción de las exportaciones de bienes manufactureros. En 2014, las exportaciones manufactureras se redujeron en alrededor 12 mil millones de dólares $(-12,8 \%)$.

En lo que respecta a la participación de los países de la región en el total de sus exportaciones, Brasil es el principal exportador. Entre 2011-2016, las exportaciones brasileras representaron en promedio más del $40 \%$ del total de las exportaciones de la región. Muy de lejos se encuentran Chile y Argentina con una participación del 13\% cada uno.

$\mathrm{Al}$ analizar las importaciones desde 2013, año donde se importó el mayor valor, se encuentra que la tasa de variación promedio fue negativa para todos los países a excepción de Bolivia. Dentro de los países que tuvieron una mayor variación negativa se encuentran Brasil (-10,5\%), Ecuador (-9,4\%) y Uruguay (-8,4\%).

De igual manera que con las exportaciones brasileñas, la menor importación de bienes manufactureros, en especial vehículos y sus partes, explico la reducción de las importaciones brasileñas. En 2014, las exportaciones manufactureras brasileñas se redujeron en alrededor de 10 mil millones de dólares (-4,3\%). Por otro lado, las importaciones ecuatorianas se redujeron debido a la implementación de sobretasas arancelarias a partir marzo de 2015. La medida en los doce primeros meses logro reducir las importaciones en un 33\%. En lo que respecta a las importaciones uruguayas, estas comenzaron a disminuir a partir de 2013 principalmente por la caída de las importaciones de combustible, hasta 2016 las importaciones de combustibles se redujeron en $55 \%$.

Además, se observa que al igual que con las exportaciones, Brasil tiene la mayor participación dentro de las importaciones de la región; representando en promedio, entre 
2013-2016, el 57\% de las importaciones de la región. Además, en segundo lugar, se encuentra también Chile (20\%) seguido muy de cerca por Argentina (19\%).

\section{Cuadro 1}

América del Sur (países seleccionados): exportaciones de bienes 2011 y 2016 (En millones de dólares y porcentajes)

\begin{tabular}{lcccc}
\hline \multicolumn{1}{c}{ País } & $\mathbf{2 0 1 1}$ & $\mathbf{2 0 1 6}$ & $\begin{array}{c}\text { Var. Prom. 2011 } \\
\mathbf{- 2 0 1 6}\end{array}$ & $\begin{array}{c}\text { Part. Prom. 2011 } \\
-\mathbf{2 0 1 6}\end{array}$ \\
\hline Argentina & 82981 & 57733 & $-6.8 \%$ & $13 \%$ \\
Bolivia & 9144 & 7082 & $-2.6 \%$ & $2 \%$ \\
Brasil & 256039 & 185235 & $-6.1 \%$ & $41 \%$ \\
Chile & 81438 & 60597 & $-5.5 \%$ & $13 \%$ \\
Colombia & 56954 & 31045 & $-10.3 \%$ & $9 \%$ \\
Ecuador & 22343 & 16798 & $-4.5 \%$ & $4 \%$ \\
Paraguay & 7776 & 8501 & $2.8 \%$ & $2 \%$ \\
Perú & 46386 & 36040 & $-4.6 \%$ & $7 \%$ \\
Uruguay & 7912 & 6964 & $-2.1 \%$ & $2 \%$ \\
\hline Total & $\mathbf{5 7 0 9 7 2}$ & $\mathbf{4 0 9 9 9 5}$ & $\mathbf{- 6 \%}$ & $\mathbf{9 2 \%}$ \\
\hline
\end{tabular}

Fuente: Elaboración propia a partir de las estadísticas de COMTRADE y TRADEMAP.

\section{Cuadro 2}

América del Sur (países seleccionados): importaciones de bienes, 2013 y 2016 (En millones de dólares y porcentajes)

\begin{tabular}{lrrcc}
\hline \multicolumn{1}{c}{ País } & $\mathbf{2 0 1 3}$ & $\mathbf{2 0 1 6}$ & $\begin{array}{r}\text { Var. Prom. } \\
\mathbf{2 0 1 3 - 2 0 1 6}\end{array}$ & $\begin{array}{r}\text { Part. Prom. } \\
\mathbf{2 0 1 3 - 2 0 1 6}\end{array}$ \\
\hline Argentina & 74442 & 55610 & $-4.5 \%$ & $18.8 \%$ \\
Bolivia & 9353 & 8427 & $0.1 \%$ & $2.8 \%$ \\
Brasil & 239748 & 137552 & $-10.5 \%$ & $57.4 \%$ \\
Chile & 79347 & 58808 & $-7.3 \%$ & $20.2 \%$ \\
Colombia & 59381 & 44831 & $-5.6 \%$ & $16.4 \%$ \\
Ecuador & 27064 & 16189 & $-9.4 \%$ & $6.8 \%$ \\
Paraguay & 12142 & 9753 & $-3.8 \%$ & $3.3 \%$ \\
Perú & 43322 & 36185 & $-3.6 \%$ & $11.8 \%$ \\
Uruguay & 11642 & 8137 & $-8.4 \%$ & $3.0 \%$ \\
\hline Total & $\mathbf{5 5 6 4 4 1}$ & $\mathbf{3 7 5} 491$ & $\mathbf{- 1 2 . 0 \%}$ & $\mathbf{9 7 . 0 \%}$ \\
\hline
\end{tabular}

Fuente: Elaboración propia a partir de las estadísticas de COMTRADE y TRADEMAP. 
En lo que respecta al número de países socios, los países sudamericanos que tuvieron mayor número de destinos para sus exportaciones en 2016 fueron Brasil, Argentina, Colombia y Chile con 220, 185, 184 y 180 países socios respectivamente. Por otro lado, los países con mayor origen de sus importaciones para 2016 fueron Brasil, Colombia y Perú con 212, 201 y 200 países socios respectivamente.

A pesar del gran número de socios comerciales que tienen los países sudamericanos, el comercio de la región se encuentra concentrado básicamente con tres socios. Entre 2007-2016, los principales socios comerciales de América del Sur han sido China, Estados Unidos y la Unión Europea quienes representaron en promedio el destino del $42 \%$ de las exportaciones y el origen del $49 \%$ de las importaciones sudamericanas. Sin embargo, su posición relativa ha variado debido a la crisis internacional que ha afectado principalmente a Estados Unidos y la Unión Europea.

En 2007, el principal socio receptor de las exportaciones de América del Sur fue la Unión Europea con 79828 millones de dólares, representando el 20\% de las exportaciones de la región. Sin embargo, debido a la recesión económica que azotó a Europa, el valor de las exportaciones a dicha región se redujo pasando a ser superadas por las exportaciones a China en 2015. Las exportaciones de Sudamérica a China tuvieron un constante incremento entre 2001 y 2013, debido principalmente al aumento de la demanda de materias primas, las cuales muchos países sudamericanos son proveedores. Sin embargo, la desaceleración de las exportaciones a esta región fue mucho menor a las experimentadas por las exportaciones a Estados Unidos y Europa. Es así que, en 2016, las exportaciones hacia China fueron de 68534 millones de dólares, representando el $17 \%$ de las exportaciones sudamericanas.

En lo que respecta a las importaciones de América del Sur hasta 2014, el principal socio fue Estados Unidos con 97269 millones de dólares, el 18\% de las importaciones sudamericanas. Sin embargo, a partir del año siguiente el principal país de origen fue China con el 20\% del total de las importaciones sudamericanas.

Los datos muestran la importancia que ha ido teniendo China en los flujos comerciales de América del Sur. Durante 2007-2010, la tasa de crecimiento de las exportaciones de la región hacia China fue más que veinte veces la tasa de crecimiento hacia el mundo. Sin embargo, cabe resaltar que dicha relevancia es mayor en el caso de las importaciones, lo cual ha significado un creciente déficit entre la región y China. Las relaciones comerciales de China con los países de la región son percibidas tanto como una oportunidad como un riesgo de reproducir el patrón de relacionamiento nortesur o centro-periferia que fue característico en las relaciones comerciales de la región durante los siglos XIX y XX (Bernal-Meza, 2016).

Si bien es cierto que China se ha convertido en el principal socio comercial para América del Sur, su importancia varía entre los países de la región. 
Gráfico 3

América del Sur: principales socios comerciales 2007-2016

(En millones de dólares)

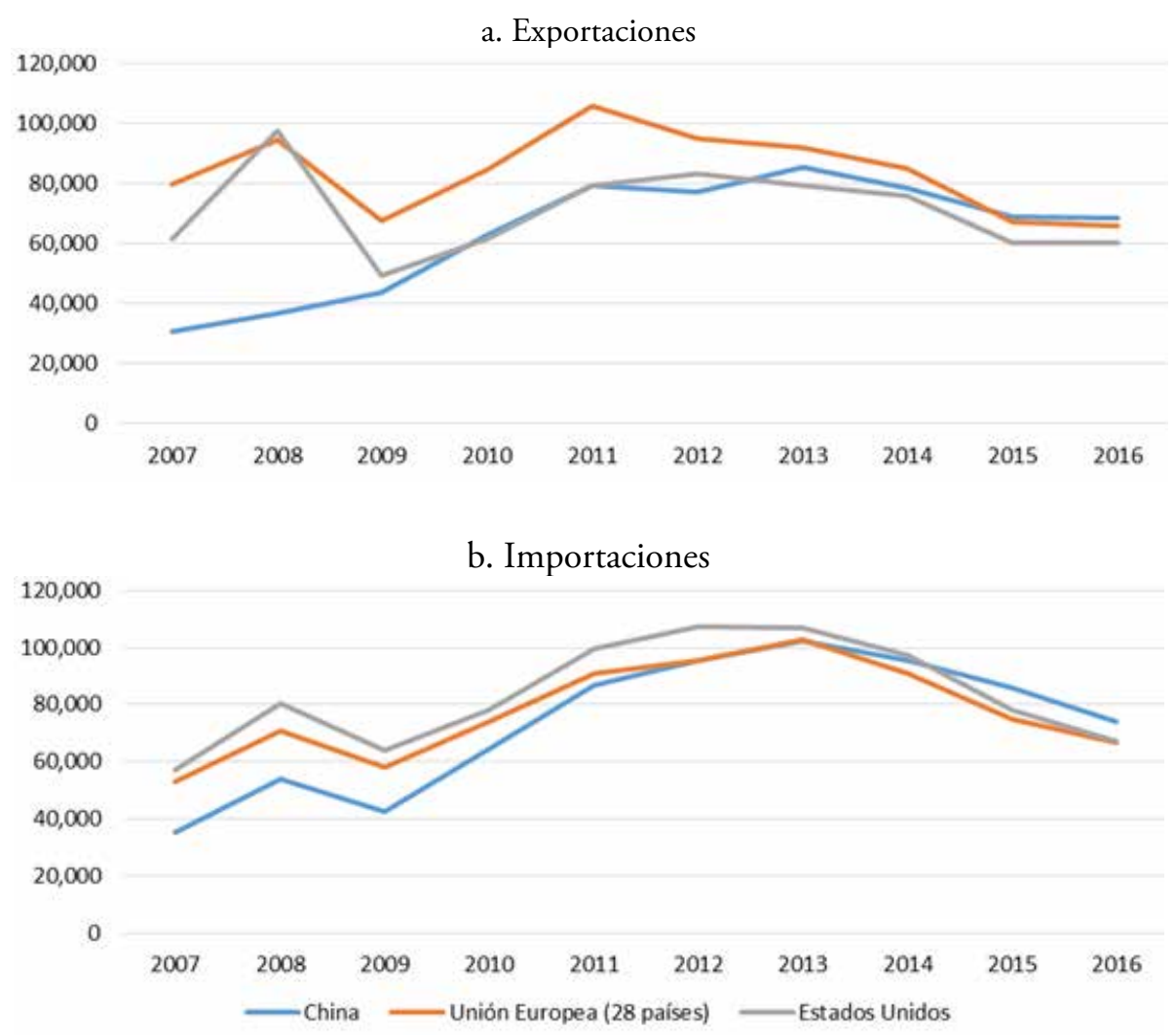

Fuente: Elaboración propia a partir de las estadísticas de COMTRADE y TRADEMAP.

En 2016, China fue el principal destino de las exportaciones de Chile, Perú y Brasil. Las exportaciones a China en los últimos años se han concentrado principalmente en cobre, hierro, semillas de soja y petróleo crudo, productos que explican el $90 \%$ de las exportaciones de cada país a dicha región, lo cual muestra un alto grado de dependencia de sus exportaciones a China (Rosales, 2016). Por otro lado, Uruguay tiene como tercer principal destino de sus exportaciones a China, a quien exporta principalmente alimentos.

En lo que respecta a las importaciones, Paraguay, Chile, Perú y Uruguay importan principalmente bienes chinos mientras que, para Argentina, Colombia y Ecuador fue su segunda principal fuente de importación y para Brasil su tercer socio principal.

A pesar de que Estados Unidos tiene en la actualidad un tercer puesto como socio comercial, es el principal socio comercial para países como Ecuador y Colombia. 
Por otro lado, como destino de las exportaciones de la región la Unión Europea se encuentra principalmente en el segundo lugar mientras como fuente de importaciones en el tercer lugar.

Los países de la región también juegan un importante papel como socios comerciales. Brasil y Argentina tienen la mayor participación en el comercio intrarregional. Brasil es el principal destino de las exportaciones de Paraguay, Bolivia, Uruguay y Argentina, mientras que es la principal fuente de las importaciones de Argentina y la segunda fuente de importaciones para Paraguay, Uruguay y Bolivia. Mientras que, Argentina es el tercer principal destino de las exportaciones de Bolivia y Paraguay, y ha sido la tercera fuente de importaciones de Paraguay.

A pesar del comercio que representa Brasil y Argentina con la región, la participación de las exportaciones sudamericanas que se dirigen a la región es muy pequeña en comparación con otras regiones como la Unión Europea o los países asiáticos. El reducido comercio intrarregional en Sudamérica se debe principalmente a su vasto tamaño, su compleja geografía, su pobre infraestructura de transporte y la similitud de productos básicos en varios países de la región (Rosales y Herreros 2017).

En los últimos años las exportaciones regionales se han vuelto menos concentradas por mercado de destino, lo cual se refleja principalmente por la reducción del peso de los Estados Unidos y la mayor importancia de China y otros mercados asiáticos (Rosales y Herreros 2017). Al analizar la concentración de las exportaciones de los países de América del Sur a través del Índice de Herfindahl y Hirschman', se encuentra que Argentina, Brasil, Paraguay y Uruguay tienen menor concentración en los destinos para sus exportaciones; mientras que Suriname, Guyana y Ecuador presentan la mayor concentración. Las economías relativamente más pequeñas muestran niveles más altos de concentración de productos.

Por otro lado, en lo que respecta a la participación en el mercado de exportaciones, los productos brasileńos tienen gran presencia dentro de las importaciones mundiales, lo cual se debe también a la mayor cantidad de productos comercializados. Entre 2007-2016, las exportaciones brasileñas representaron más el 1\% de las exportaciones mundiales.

\footnotetext{
1 El índice de Herfindahl y Hirschman $(\mathrm{HH})$ es una medida que informa sobre la concentración económica de un mercado. Un valor próximo a 1 indica que el comercio de un país está concentrado en muy pocos mercados, mientras que un valor cercano a 0 indica que un país tiene una cartera comercial muy diversificada.
} 
Cuadro 3

América del Sur (países seleccionados)

Principales socios comerciales para cada país (2016)

a. Exportaciones

\begin{tabular}{|c|c|c|c|c|c|c|}
\hline & $1^{\circ}$ & $\%$ & $2^{\circ}$ & $\%$ & $3^{\circ}$ & $\%$ \\
\hline Argentina & Brasil & 15,6 & Unión Europea & 14,8 & Estados Unidos & 7,8 \\
\hline Bolivia & Brasil & 19,2 & Estados Unidos & 13,6 & Argentina & 11,4 \\
\hline Brasil & China & 19,0 & Unión Europea & 18,0 & Estados Unidos & 12,6 \\
\hline Chile & China & 28,5 & Estados Unidos & 14,1 & Unión Europea & 12,6 \\
\hline Colombia & Estados Unidos & 32,9 & Unión Europea & 16,1 & Panamá & 6,2 \\
\hline Ecuador & Estados Unidos & 32,4 & Unión Europea & 16,9 & Chile & 6,8 \\
\hline Paraguay & Brasil & 35,4 & Unión Europea & 14,4 & Argentina & 10,0 \\
\hline Perú & China & 23,5 & Estados Unidos & 17,3 & Unión Europea & 15,3 \\
\hline Uruguay & Brasil & 17,2 & Unión Europea & 13,0 & China & 12,8 \\
\hline \multicolumn{7}{|c|}{ b. Importaciones } \\
\hline & $1^{\circ}$ & $\%$ & $2^{\circ}$ & $\%$ & $3^{\circ}$ & $\%$ \\
\hline Argentina & Brasil & 24,5 & China & 18,8 & Estados Unidos & 12,6 \\
\hline Bolivia & China & 20,0 & Brasil & 17,6 & Unión Europea & 10,6 \\
\hline Brasil & Unión Europea & 22,6 & Estados Unidos & 17,5 & China & 17,0 \\
\hline Chile & China & 24,1 & Estados Unidos & 17,4 & Unión Europea & 17,1 \\
\hline Colombia & Estados Unidos & 26,7 & China & 19,3 & Unión Europea & 14,0 \\
\hline Ecuador & Estados Unidos & 23,2 & China & 19,1 & Unión Europea & 11,6 \\
\hline Paraguay & China & 27,1 & Brasil & 24,2 & Argentina & 14,7 \\
\hline Perú & China & 22,8 & Estados Unidos & 19,6 & Unión Europea & 12,1 \\
\hline Uruguay & China & 18,8 & Brasil & 18,0 & Unión Europea & 18,0 \\
\hline
\end{tabular}

Fuente: Elaboración propia a partir de las estadísticas de COMTRADE.

En lo que concierne al número de productos comercializados ${ }^{2}$, el número de productos importados es superior a los exportados. En promedio los países de la región exportaron aproximadamente 2670 productos, mientras que importan más de 4000 productos. Brasil exportó en promedio entre 2014-2016, 4046 productos, siendo el país de la región con mayor número de productos exportados. Mientras que, Chile fue el país con mayor cantidad de productos importados, 4325 .

2 Respecto con la clasificación S.A. a 6 dígitos. 


\begin{tabular}{lll}
$\begin{array}{c}\text { Cuadro } 4 \\
\text { América Latina. Índice de Herfindahl e Hirschman (IHH) }\end{array}$ \\
Índice de penetración en el mercado de exportación (IPME) \\
\cline { 2 - 3 } País & IHH & IPME \\
\hline Argentina & 0.05 & 5.34 \\
Bolivia & 0.14 & 2.06 \\
Brasil & 0.09 & 11.3 \\
Chile & 0.13 & 4.48 \\
Colombia & 0.18 & 5.16 \\
Ecuador & 0.15 & 2.87 \\
Guyana & 0.23 & 1.36 \\
Paraguay & 0.08 & 1.81 \\
Perú & 0.11 & 4.41 \\
Suriname & 0.20 & 1.46 \\
Uruguay & 0.09 & 2.54 \\
\hline
\end{tabular}

Fuente: Elaboración propia a partir de datos estadísticos de World Integrated Trade Solution (WITS) (Banco Mundial, 2017).

\section{Cuadro 5}

América del Sur (países seleccionados)

Número de productos comercializados, promedio 2014-2016

\begin{tabular}{lcc}
\hline País & $\mathrm{N}^{\circ}$ Productos exportados & $\mathrm{N}^{\circ}$ Productos importados \\
\hline Argentina & 3400 & 4032 \\
Bolivia & 758 & 3981 \\
Brasil & 4046 & 4313 \\
Chile & 3528 & 4325 \\
Colombia & 3441 & 4262 \\
Ecuador & 2389 & 4013 \\
Paraguay & 1346 & 3730 \\
Perú & 3289 & 4150 \\
Uruguay & 1829 & 3935 \\
\hline
\end{tabular}

Fuente: Elaboración propia a partir de datos estadísticos de World Integrated Trade Solution (WITS) (Banco Mundial, 2017).

Nota: Realizado utilizando la Clasificación S.A. a 6 dígitos. El promedio de Bolivia es de 2015 y 2016.

3 El Índice de penetración en el mercado de exportación es una medida que informa sobre la relación entre el número de países a los que exporta cierto producto el país declarante con el número de países que importaron dicho producto. 
Sudamérica ha tenido tradicionalmente ventaja comparativa en las exportaciones de combustibles, minerales y otros productos primarios, quedando rezagada en las exportaciones de productos manufacturados intensivos en habilidades y tecnología. A pesar de que hubo diferentes intentos en el siglo pasado, como políticas de sustitución de importaciones entre otras, para desarrollar otros segmentos de la economía, el auge de los precios de los productos básicos en la década de 2000 mermó dichos esfuerzos, debido a que los productos primarios menos complejos de la región representaron una mayor participación de las exportaciones. En este sentido al tener un sector exportador primario, la región queda vulnerable ante cambios en los patrones de demanda y de los precios internacionales.

Entre 2007-2016, las exportaciones de materias primas significaron en promedio más del $40 \%$ de las exportaciones totales, seguida de las exportaciones de bienes intermedios, bienes de consumo y bienes de capital que representaron el $30 \%, 17 \%$ y $6 \%$ respectivamente del total de las exportaciones.

$\mathrm{Al}$ analizar las exportaciones por nivel de procesamiento para cada país, entre 2007 y 2016, se encontró que Bolivia exportó principalmente bienes de consumo, siendo aproximadamente el $48 \%$ de sus exportaciones; mientras que Paraguay, Perú y Argentina exportaron principalmente bienes intermedios, alrededor del 48\%, 43\% y $41 \%$ de sus exportaciones respectivamente. Por otro lado, Ecuador, Colombia, Uruguay, Chile y Brasil exportaron principalmente materias primas, 72\%, 57\%, $50 \%, 39 \%$ y $36 \%$ de sus exportaciones respectivamente.

Brasil al tener la mayor participación en las exportaciones de la región tiene también la mayor participación en las exportaciones para cada nivel de procesamiento. Con lo cual, las exportaciones brasileras de bienes de capital representaron en promedio entre 2007-2016 alrededor del 75\% de las exportaciones de bienes de capital de la región, cifra muy superior a su participación en las exportaciones regionales. Por otro lado, en lo que respecta a las exportaciones de bienes intermedios y materias primas de la región, Brasil representó alrededor del 37\% para los respectivos niveles de procesamiento, mientras que sus exportaciones de bienes de consumo representaron el 35\%.

Por otro lado, al tener Sudamérica una industria muy poca desarrollada, principalmente importa bienes de capital, los cuales entre 2007-2006 representaron el 33\% de sus importaciones. Asimismo, los bienes de consumo e intermedios tienen una alta participación en las importaciones de la región al representar el 31\% y $25 \%$ respectivamente. Por otro lado, al ser Sudamérica un país altamente proveedor de materias primas, el porcentaje de estas que importa es pequeño, tan solo del $10 \%$. 


\section{Gráfico 4}

América del Sur: exportaciones por nivel de procesamiento

(Participación en \%)

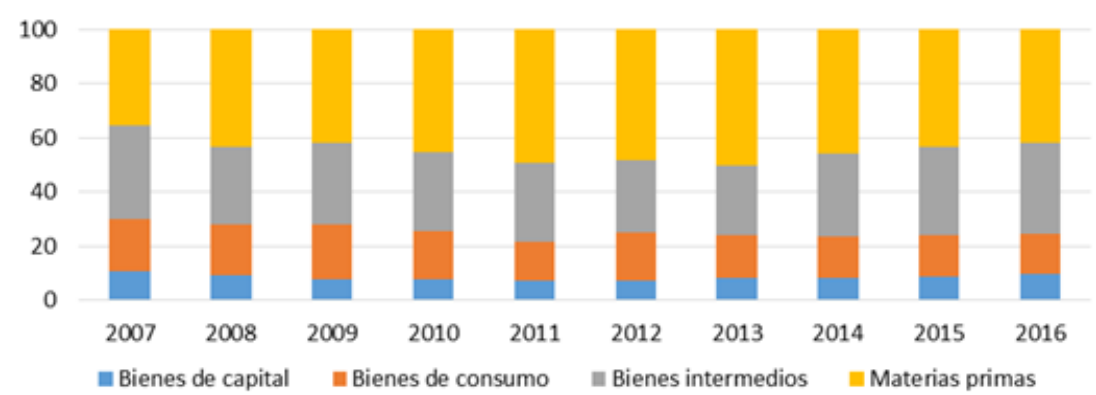

Fuente: Elaboración propia a partir de datos estadísticos de World Integrated Trade Solution (WITS) (Banco Mundial, 2017).

Nota: No se tienen datos disponibles para Venezuela (2014-2016), para Suriname (2015) y para Bolivia (2016).

\section{Gráfico 5}

América del Sur: importaciones por nivel de procesamiento (Participación en \%)

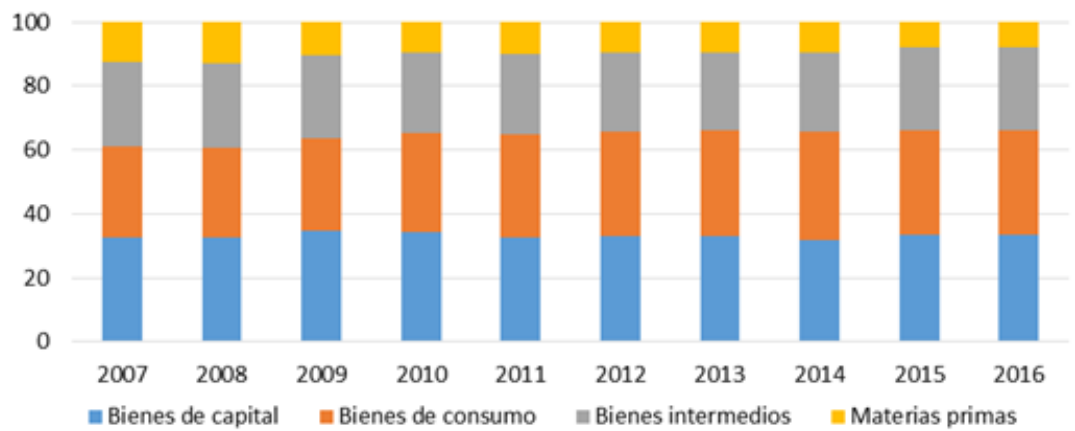

Fuente: Elaboración propia a partir de datos estadísticos de World Integrated Trade Solution (wits) (Banco Mundial, 2017).

Nota: No se tienen datos disponibles para Venezuela (2014-2016), para Suriname (2015) y para Bolivia (2016).

En lo que respecta a los principales productos comercializados, se encuentra una alta concentración entre los cinco primeros productos comercializados, en especial para el caso de las exportaciones.

Las exportaciones de los cinco principales productos en 2016 representaron en promedio más del 55\% del total de las exportaciones de la región; sin embargo, dicho porcentaje varía de acuerdo con los países. Los cinco principales productos para Brasil representaron el 32\% del total de sus exportaciones mientras que para Ecuador fue el $72 \%$. 
$\mathrm{Al}$ analizar los cinco principales productos exportados destacan productos agrícolas, ganaderos, minerales e hidrocarburos. Los países del noroeste exportan principalmente petróleo representando entre el $30 \%$ y $25 \%$ de sus exportaciones. Por otro lado, Chile y Perú exportan principalmente cobre, siendo los principales proveedores de este mineral con el $40 \%$ de participación en las exportaciones mundiales. Además, Brasil y Argentina tuvieron como principal producto exportado a la soja, representando el 50\% de las exportaciones mundiales de producto. Mientras que Uruguay exportó principalmente carne, cuyas exportaciones tuvieron como principal destino a China.

Principalmente los productos exportados son materias primas, sin embargo, también se encuentra el caso de exportaciones de manufacturas industriales como es el caso de los vehículos para el transporte de mercancías exportados por Argentina. Alrededor del 85\% de las exportaciones de vehículos producidos en Argentina tienen como destino Brasil.

Por otro lado, a diferencia de las exportaciones, el promedio de los cinco principales productos representa una menor proporción del total comerciado, siendo este el $22 \%$. Dentro de los principales productos destacan los hidrocarburos, medicamentos, vehículos y aparatos telefónicos.

El principal producto importado para todos los países de la región a excepción de Argentina fue petróleo representando entre el 11\% y 6\% de las importaciones de cada país. Mientras que, para Argentina, el principal producto importado fue vehículos con el $8 \%$.

Las exportaciones e importaciones se han reducido en los últimos ańos de estudio debido a los cambios en los precios internacionales, la crisis internacional y la desaceleración china. Dicho contexto internacional afecto la posición de los diferentes socios comerciales de Sudamérica, aumentado la participación comercial de China.

Por otro lado, dentro de los principales productos exportados destacan las materias primas, dentro de ellas el cobre, la soja y el petróleo. En lo que respecta a las importaciones, ante poca desarrollada industria Sudamérica, dentro de los principales productos importados destacan bienes de capital, consumo e intermedio como vehículos, aparatos telefónicos, medicamentos e hidrocarburos.

\section{Comercio de servicios}

El comercio de servicios se ha incrementado a nivel mundial debido a la difusión de las tecnologías de la información y las comunicaciones (TIC), que elimino los limites tradicionales entre los bienes y los servicios, y la importancia clave que tienen diferentes servicios en la operación de las cadenas de valor de bienes como es el caso de la investigación y desarrollo (I+D), los servicios financieros y logísticos y la mercadotecnia (Cepal, 2017). 


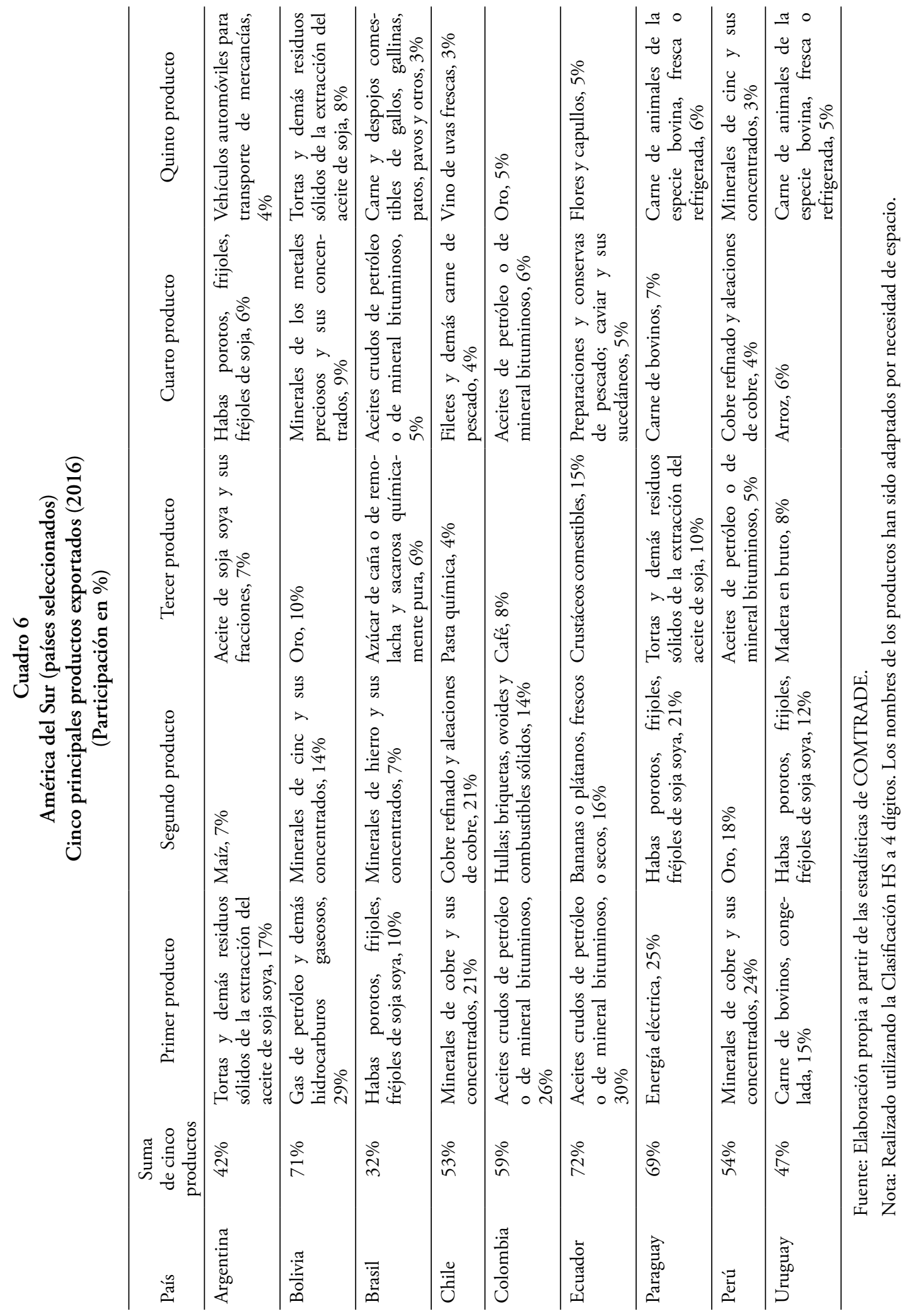




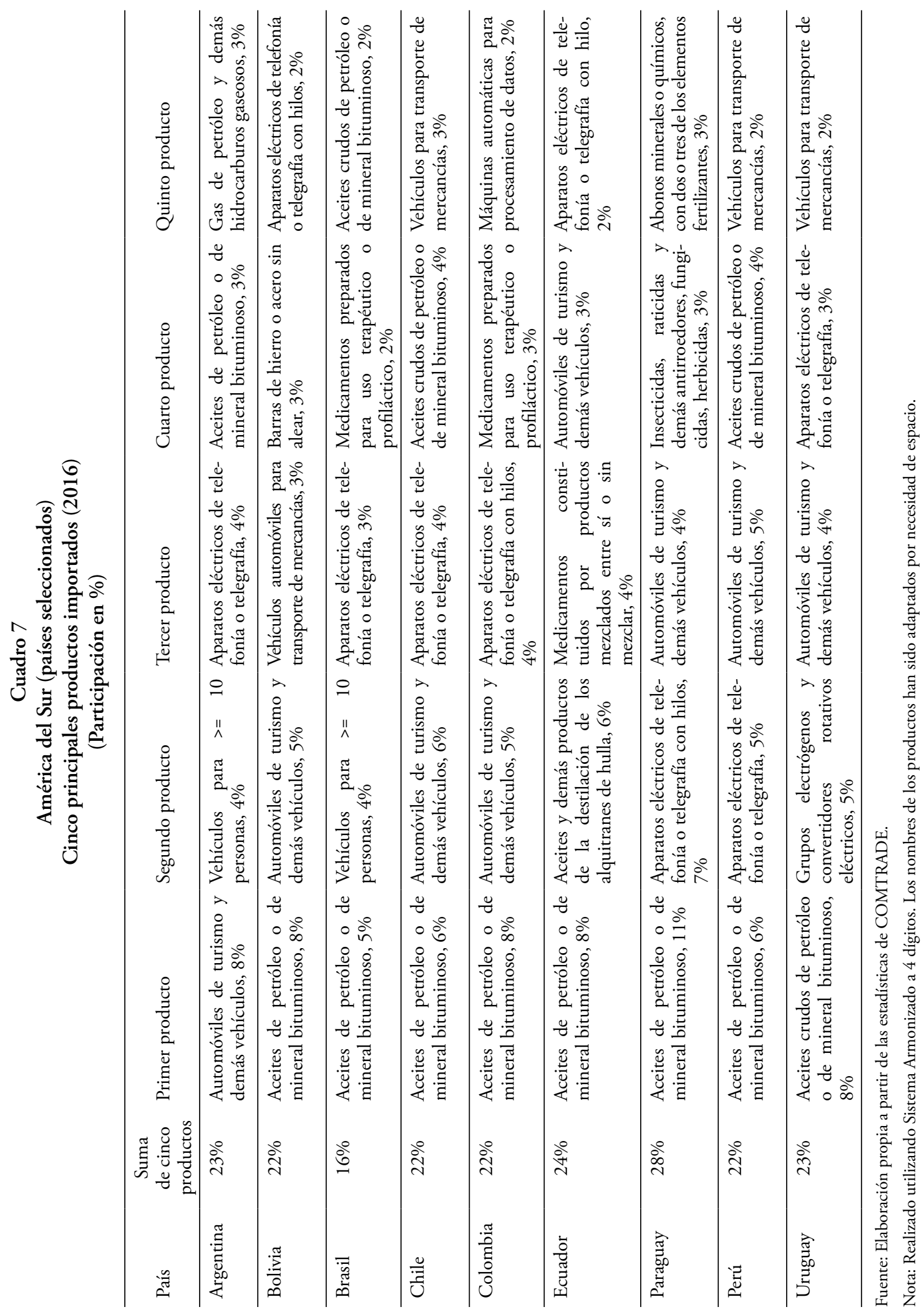


Las exportaciones de servicios de la región crecieron igual que la mundial en 4,3\% en el periodo 2007-2016, mientras que las importaciones de la región lo hicieron a una tasa de dos puntos porcentuales más alta que la tasa mundial $(4,3 \%)$.

En 2012, se alcanzó el mayor valor de las exportaciones con 87848 millones de dólares, mientras que las importaciones fueron las más altas en 2013 con 169191 millones de dólares. La región ha presentado déficits en el comercio de servicios, reduciéndose en los dos últimos ańos debido a la menor importación que se explica por la menor importación de servicios de transporte (debido al menor comercio de mercancías) y la reducción del turismo hacia el exterior.

En lo que respecta a la participación de la región en el comercio mundial de servicios, las exportaciones de servicios de la región representaron entre 2007 y 2016 menos del $2 \%$, mientras que las importaciones de la región representaron en promedio el 3,2\%.

La información sobre los socios comerciales es muy escasa, sin embargo, se tiene la información para Brasil, Chile y Colombia. De donde se rescata la importancia del mercado regional para las exportaciones e importaciones de Chile y Colombia, representando más del $40 \%$ de sus exportaciones y más del $20 \%$ de sus importaciones. Mientras que, para Brasil, el principal destino de sus exportaciones es la Unión Europea con más del $30 \%$ y también de sus importaciones con más del $40 \%$.

El mayor comercializador de servicios al igual que bienes ha sido Brasil, sus exportaciones fueron de 32930 millones de dólares en 2016, siendo más del 40\% de las exportaciones de la región. A pesar de su importante participación a nivel regional, a nivel mundial no tiene una importante posición quedando rezagado al puesto 32 . Asimismo, destaca Argentina con más del 15\% de participación, con la posición 50 a nivel mundial. Por otro lado, es importante señalar, que los únicos países que han incrementado sus exportaciones en los últimos tres años son Colombia y Perú.

\section{Gráfico 6}

América del Sur: comercio de servicios, 2007-2016

\section{(En millones de dólares)}

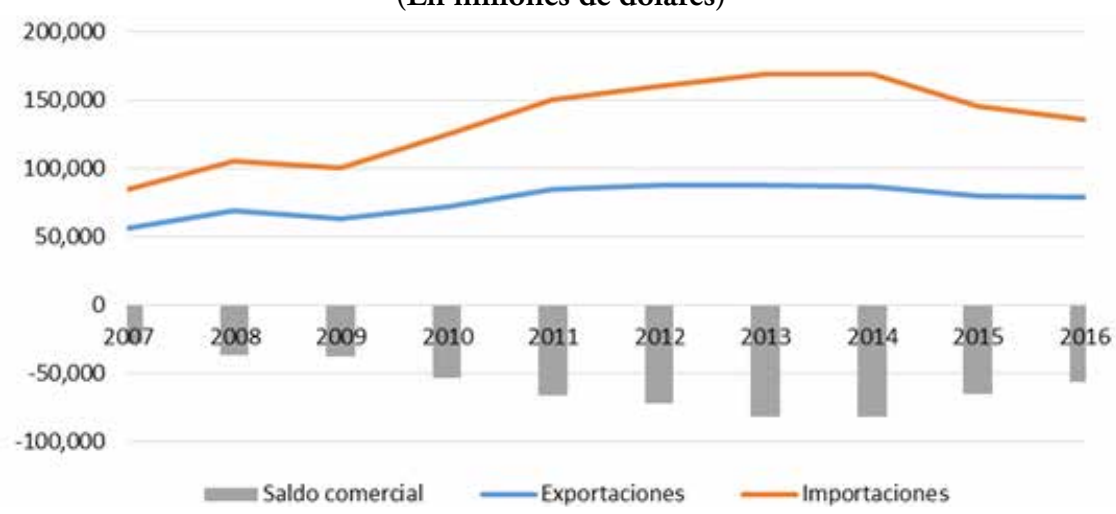

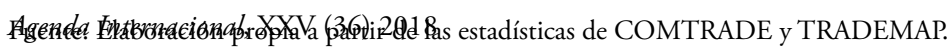




\section{Gráfico 7}

América del Sur: participación en el comercio internacional de servicios, 2007-2016

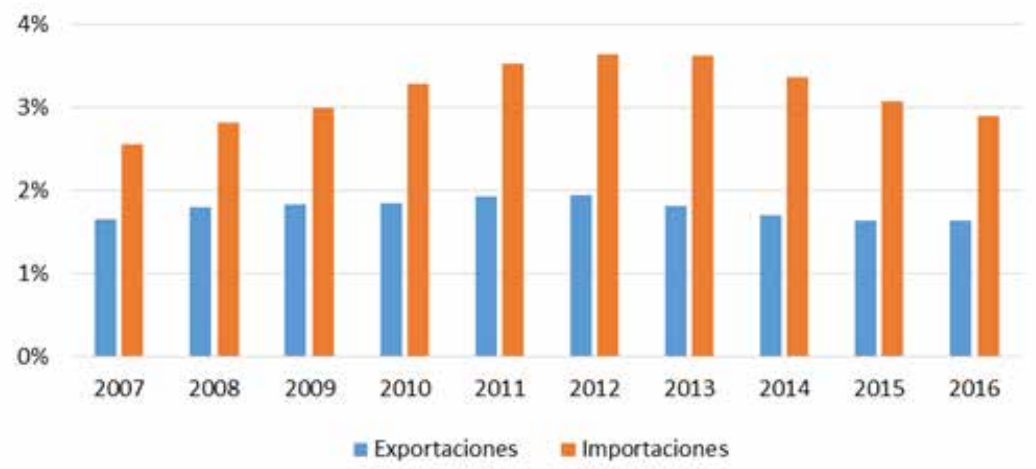

Fuente: Elaboración propia a partir de las estadísticas de COMTRADE y TRADEMAP.

\section{Cuadro 8}

América del sur (países seleccionados)

Exportaciones de servicios, 2014-2016

(En millones de dólares y porcentajes)

\begin{tabular}{lccccc}
\hline País & $\mathbf{2 0 1 4}$ & $\mathbf{2 0 1 5}$ & $\mathbf{2 0 1 6}$ & $\begin{array}{c}\text { Var. Prom. } \\
\mathbf{2 0 1 5 - 2 0 1 6}\end{array}$ & $\begin{array}{c}\text { Part. Prom. } \\
\mathbf{2 0 1 6}\end{array}$ \\
\hline Argentina & 13842 & 13917 & 12655 & $-9,1 \%$ & $16,0 \%$ \\
Bolivia & 1242 & 1149 & 1209 & $5,2 \%$ & $1,5 \%$ \\
Brasil & 39592 & 33328 & 32930 & $-1,2 \%$ & $41,5 \%$ \\
Chile & 10967 & 9777 & 9500 & $-2,8 \%$ & $12,0 \%$ \\
Colombia & 6854 & 7117 & 7980 & $12,1 \%$ & $10,1 \%$ \\
Ecuador & 2346 & 2391 & 2140 & $-10,5 \%$ & $2,7 \%$ \\
Paraguay & 892 & 859 & 965 & $12,4 \%$ & $1,2 \%$ \\
Perú & 5874 & 6226 & 6304 & $1,2 \%$ & $7,9 \%$ \\
Uruguay & 3345 & 3003 & 4148 & $38,1 \%$ & $5,2 \%$ \\
Venezuela & 1869 & 1589 & 1281 & $-19,4 \%$ & $1,6 \%$ \\
Total & 86823 & 79356 & 79113 & $-4,6 \%$ & $99,8 \%$ \\
\hline
\end{tabular}

Fuente: Elaboración propia a partir de las estadísticas de COMTRADE y TRADEMAP.

En lo que respecta a las importaciones de servicios, Brasil es el principal importador dentro de la región, con la posición número 22 a nivel mundial. Las importaciones de los países de la región han disminuido en los últimos años a excepción del caso de Argentina y Uruguay que presentaron un importante crecimiento para 2016. 
Al analizar el déficit por países solo Uruguay presentó superávit en 2016, mientras que el resto de países presentaron déficits, siendo Brasil el de mayor déficit comercial. Sin embargo, al analizar el déficit proporcional a su PBI, se encuentra que Bolivia tiene el mayor déficit en el comercio de servicios (5\% de su PBI) tanto en servicios modernos ${ }^{4}$ como en tradicionales 5 . Por otro lado, otros países con representativos saldos comerciales como porcentaje de su PBI son Chile, Brasil, explicado por el déficit en el comercio de servicios modernos, y Argentina, por el déficit en el comercio de servicios tradicionales (Cepal, 2017).

Por otro lado, al desagregar las balanzas comerciales por categorías, se encuentra que las principales categorías con mayor déficit comercial son los servicios de transporte, otros servicios empresariales, propiedad intelectual y viajes.

\section{Cuadro 9}

América del Sur (países seleccionados)

Exportaciones de servicios, 2014-2016

(En millones de dólares y porcentajes)

\begin{tabular}{lccccc}
\hline \multicolumn{1}{c}{ País } & $\mathbf{2 0 1 4}$ & $\mathbf{2 0 1 5}$ & $\mathbf{2 0 1 6}$ & $\begin{array}{c}\text { Var. Prom. } \\
\mathbf{2 0 1 5 - 2 0 1 6}\end{array}$ & $\begin{array}{c}\text { Part. Prom. } \\
\mathbf{2 0 1 6}\end{array}$ \\
\hline Argentina & 17002 & 17841 & 21049 & $18,0 \%$ & $15,5 \%$ \\
Bolivia & 3040 & 2317 & 2281 & $-1,6 \%$ & $1,7 \%$ \\
Brasil & 87873 & 70500 & 63565 & $-9,8 \%$ & $46,8 \%$ \\
Chile & 14724 & 13589 & 12638 & $-7,0 \%$ & $9,3 \%$ \\
Colombia & 12945 & 10762 & 10872 & $1,0 \%$ & $8,0 \%$ \\
Ecuador & 3517 & 3197 & 3195 & $0,0 \%$ & $2,4 \%$ \\
Paraguay & 1114 & 1104 & 1093 & $-1,0 \%$ & $0,8 \%$ \\
Perú & 7674 & 7958 & 7956 & $0,0 \%$ & $5,9 \%$ \\
Uruguay & 3206 & 2647 & 3303 & $24,8 \%$ & $2,4 \%$ \\
Venezuela & 16884 & 14268 & 9440 & $-33,8 \%$ & $6,9 \%$ \\
\multicolumn{1}{c}{ Total } & 167979 & 144184 & 135391 & $-10,1 \%$ & $99,7 \%$ \\
\hline
\end{tabular}

Fuente: Elaboración propia a partir de las estadísticas de COMTRADE y TRADEMAP.

Al igual que en el comercio mundial de servicios, en Sudamérica, el comercio de servicios tradicionales como los servicios de transporte y viajes tienen una importante participación en el comercio regional.

\footnotetext{
4 Se refiere a los servicios de telecomunicaciones, servicios de informática e información, servicios financieros, servicios de seguros y pensiones regalías y otros servicios empresariales.

5 Se refiere a servicios relacionados con los bienes, transporte, viajes, construcción, servicios personales, culturales y recreativos y servicios gubernamentales.
} 
La exportación de servicios de transporte tiene un gran porcentaje de participación dentro de las exportaciones de Paraguay y Venezuela con más del 40\%, sin embargo, el principal exportador de la región es Brasil con 5058 millones de dólares en 2016. Los servicios de transporte principalmente exportados son por vía marítima y área, Brasil es el principal exportador de servicios de transporte por vía marítima mientras que Colombia es el principal exportador de servicios de transporte por vía aérea.

En lo que respecta a los servicios de viajes, estos tienen una importante participación dentro de la canasta de exportaciones de servicios para Bolivia, Colombia, Ecuador, Perú y Uruguay.

Además, dentro de los principales exportadores de servicios de tecnología de la información y comunicaciones destacan Brasil con aproximadamente 1800 millones de dólares y Argentina con más de 1400 millones de dólares. Si bien los países de América del Sur tienen una participación limitada dentro del comercio mundial de servicios, hay casos exitosos en que se ha logrado consolidar ventajas comparativas y se ha penetrado en mercados dinámicos dentro del mundo de los servicios intensivos en conocimiento (López, Niembro y Ramos, 2014).

En 2016, los servicios personales y culturales tuvieron una importante participación en las exportaciones de Argentina (222 millones de dólares) y Colombia (129 millones de dólares) encontrándose dentro de los cinco principales servicios exportados. Por otro lado, los servicios de seguro y pensiones representaron un importante papel dentro de las exportaciones brasileras siendo alrededor de 1650 millones de dólares.

\author{
Gráfico 8 \\ América del Sur (países seleccionados) \\ Exportaciones de servicios por tipo de servicios, 2016 \\ (En porcentajes)
}

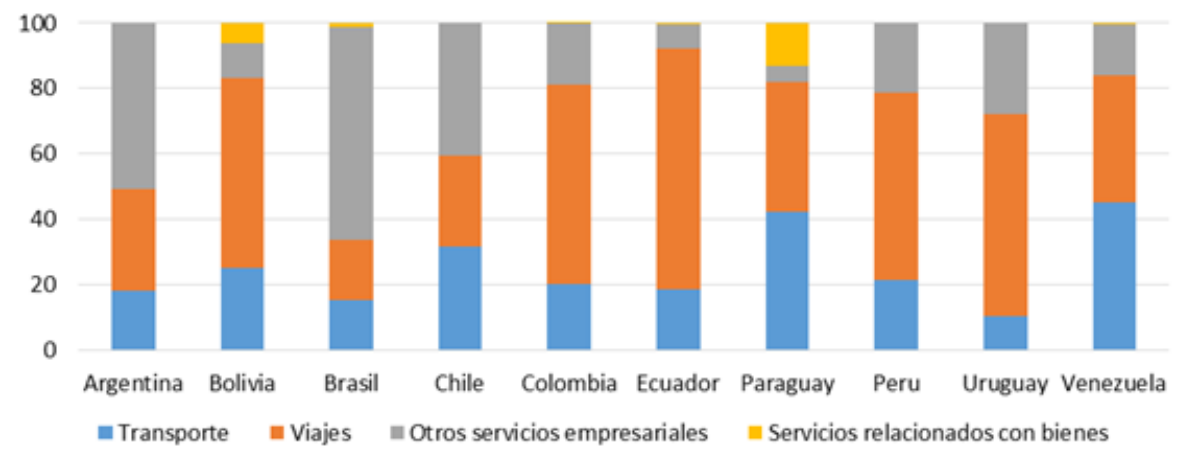

Fuente: Elaboración propia a partir de las estadísticas de la Organización Mundial del Comercio (OMC). 
Las importaciones de servicios de transporte y viajes representan más del $50 \%$ del total de importaciones de servicios para la mayoría de países de la región a excepción de Brasil y Venezuela quienes importan otros servicios comerciales.

\section{Gráfico 9}

América del Sur (países seleccionados):

Importaciones de servicios por tipo de servicios, 2016

(En porcentajes)

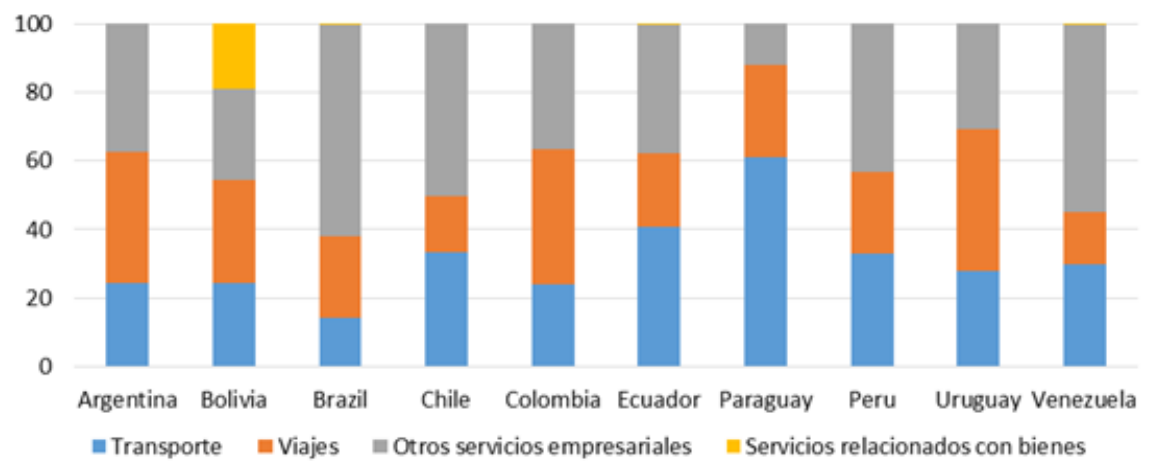

Fuente: Elaboración propia a partir de las estadísticas de la Organización Mundial del Comercio (OMC).

El comercio de servicios de la región se ha caracterizado por deficitario. El principal exportador e importador de la región es Brasil, aunque en las exportaciones mundiales no tiene una importante posición. Además, tanto por el lado de las exportaciones como importaciones los servicios tradicionales son los más importantes.

\section{Conclusiones}

Los países de América del Sur aprovecharon el buen contexto internacional hasta el 2008; luego, el comercio de la región se vio impulsado principalmente por el crecimiento económico chino y el aumento de los precios de las materias primas debido a la mayor demanda de este último. Sin embargo, a partir de la desaceleración china y la caída de los precios de los commodities, el valor comerciado por la región fue reduciéndose.

La participación de la región dentro del comercio mundial alcanzó su máximo en 2011, luego se reduciría hasta representar 2,6\% de las exportaciones mundiales de bienes y $1,6 \%$ de las exportaciones mundiales de servicios.

América del Sur exporta principalmente materias primas como productos agrícolas, minerales e hidrocarburos mientras que importa principalmente bienes de capital 
consumo e intermedios. Dentro de los principales productos importados destacan hidrocarburos, medicamentos, vehículos y aparatos telefónicos.

En lo que respecta al comercio de servicios, Brasil también juega el rol principal. Los países de la región exportan principalmente servicios tradicionales como transporte y viajes. Por otro lado, Brasil y Argentina comercian un importante valor de servicios modernos como los servicios relacionados con tecnología de la información y comunicación.

\section{Referencias}

Banco Mundial. (2017). WITS World Integrated Trade Solution. En: https://wits.worldbank.org Bartesaghi, I. (2013). El desempeño económico y comercial de América Latina y el Caribe en la última década. Montevideo: Departamento de Negocios Internacionales e Integración. Facultad de Ciencias Empresariales. Universidad Católica del Uruguay. Recuperado de https://ucu.edu.uy/sites/default/files/pdf/2015/desempeno_economico_comercial_america_latina_caribe_ultima_decada.pdf

Bernal-Meza, R. (2016). China y América Latina: de la oportunidad al desafío. Revista Tempo do Mundo, 2(2). Recuperado de http://repositorio.ipea.gov.br/bitstream/11058/6542/1/ TdM_v2_n2_China.pdf

Comisión Económica para América Latina y el Caribe (Cepal). (2012). Boletín Estadístico Comercio Exterior de Bienes en América Latina y el Caribe, 6. Recuperado de https:// www.cepal.org/es/publicaciones/40313-boletin-estadistico-comercio-exterior-bienesamerica-latina-caribe-primer

Comisión Económica para América Latina y el Caribe (Cepal). (2013). Boletín Estadístico Comercio Exterior de Bienes en América Latina y el Caribe, 10. Recuperado de https:// www.cepal.org/es/publicaciones/35959-boletin-estadistico-comercio-exterior-bienesamerica-latina-caribe-primer

Comisión Económica para América Latina y el Caribe (Cepal). (2015). Boletín Estadístico Comercio Exterior de Bienes en América Latina y el Caribe, 18. Recuperado de https:// www.cepal.org/es/publicaciones/38327-boletin-estadistico-comercio-exterior-bienesamerica-latina-caribe-primer

Comisión Económica para América Latina y el Caribe (Cepal). (2017). Perspectivas del Comercio Internacional de América Latina y el Caribe. Recuperación en un contexto de incertidumbre. Santiago de Chile: Naciones Unidas. Recuperado de https://repositorio.cepal.org/handle/11362/42315

COMTRADE (2017). United Nations commodity trade statistics database. Naciones Unidas. Recuperado de https://comtrade.un.org/

FMI - Fondo Monetario Internacional. (2017). Cluster Report: Trade Integration in Latin America and the Caribbean. Country Report No. 17/66. Recuperado de https://www.imf.org/en/Publications/CR/Issues/2017/03/10/Cluster-ReportTrade-Integration-in-Latin-America-and-the-Caribbean-44735 
López, A., Niembro, A. y Ramos, D. (2014). La competitividad de América Latina en el comercio de servicios basados en el conocimiento. Revista Cepal, 113, 24-41. Recuperado de http://www20.iadb.org/intal/catalogo/PE/2014/14606.pdf_

Map, T. (2017). Trade statistics for international business development. Recuperado de https:// www.trademap.org

OMC. (2017). Trade profiles. Recuperado de http://stat.wto.org/Home/WSDBHome. aspx?Language $=\mathrm{E}$

Roldán, A., Castro, A., Pérez Restrepo, C., Echavarría, P. y Evan, R. (2016). La presencia de China en América Latina: Comercio, inversión y cooperación económica. Recuperado de http://www.eafit.edu.co

Rosales, O. y Herreros, S. (2017). Desafíos de la competitividad exportadora en América Latina y el Caribe. Estudios Internacionales, 49, 125-141. https://doi. org/10.5354/0719-3769.2017.47536

Vargas, L. M. (2017). Caracterización del comercio exterior colombiano que exporta realmente Colombia. Medellín: Facultad de Ciencias Administrativas y Económicas del Tecnológico de Antioquia Institución Universitaria. Recuperado de http://dspace. tdea.edu.co/

Fecha de recepción: 16 de marzo de 2018

Fecha de aceptación: 25 de junio de 2018 